\title{
A new quantitative assay for cytochrome $c$ release in apoptotic cells
}

\author{
Cell Death and Differentiation (2003) 10, 853-855. doi: 10.1038/sj.cdd.4401263
}

Dear Editor,

Selective permeabilization of the mitochondrial outer membrane is an integral event in apoptosis induced by numerous stimuli. ${ }^{1,2}$ As a result, several proapoptotic proteins including cytochrome $c$ are released from the mitochondrial intermembrane space to the cytoplasm. Cytochrome $c$ then triggers activation of caspase proteases via the formation of a complex known as apoptosome. ${ }^{3}$ Current techniques to assay cytochrome $c$ release rely on cellular fractionation followed by Western blotting, immunocytochemistry or the subcellular localization of GFP-tagged cytochrome $c .{ }^{4}$ These techniques have inherent problems that make it difficult to accurately quantitate the number of cells in which cytochrome $c$ has translocated. In this letter, we highlight some of the problems associated with current methods to follow cytochrome $c$ release (Table 1) and suggest an adaptation of current protocols to quantitate the number of cells with cytoplasmic cytochrome $c$ in both adherent and nonadherent cell populations.

Owing to the relative abundance of cytochrome $c$ within cells (estimated between 0.5 and $5 \mathrm{mM}$ in the intermembrane space), Western blotting of cellular fractions is a useful technique to observe gross changes in cytochrome $c$ distribution within cells. It is of some concern however that, due to the abundance of cytochrome $c$, small changes in overall cellular distribution may appear substantial if only cytosolic fractions are assayed (Figure 1ai). Studies that assay both the mitochondrial and cytosolic fractions give a more representative analysis of the extent of cytochrome $c$ release (Figure 1aii). Using Western blotting, it is often difficult to expose X-ray film such that the bands from control cell populations (often $5 \%$ apoptosis) and apoptotic populations (where most of the cells have cytoplasmic cytochrome $c$ ) are both within the linear range (i.e. quantifiable on a densitometer). It is therefore difficult to use Western blotting for accurate quantitative analysis. This problem is compounded by the fact that cytoplasmic cytochrome $c$ may leak out of cells that have deviated to secondary necrosis (as often occurs several hours after the onset of apoptosis in long-term assays). It is also not ideal that Western blotting shows an averaged result from a population of cells. It is therefore not possible to determine whether all the cytochrome $c$ is cytoplasmic in a small percentage of cells or all cells have partially redistributed their cytochrome $c$.

Immunocytochemistry followed by fluorescence microscopy has been invaluable in quantitating the number of cells that have punctate or diffuse (released) distribution of cytochrome $c$. This data, however, is frequently not quantitated since counting the cells is laborious. Further, it is often difficult to determine whether cells in suspension or that have rounded up, have punctate or diffuse staining. Cells that have undergone secondary necrosis may have lost much of their staining and may be ignored, unless they are arbitrarily categorized as dead.

Expression of GFP-tagged cytochrome has made it possible to follow cytochrome $c$ redistribution within cells in real time. Using these cells in combination with selective permeabilization of the plasma membrane by digitonin, it is possible to use FACS analysis to rapidly determine the number of cells in which cyochrome $c$ has translocated to the cytoplasm. ${ }^{5}$ This assay is based on the idea that permeabilization of cells will allow cytoplasmic cytochrome $c$-GFP to diffuse out of the cells. Cells with cytoplasmic cytochrome $c$ GFP will therefore have less GFP fluorescence than cells with intact mitochondria. This method, however, is only useful for cells that express GFP-cytochrome $c$. We reasoned that this assay could be coupled with immunocytochemistry (outlined in Figure $1 \mathrm{~b}$ and the method below) to quantitate the percentage of cells that have cytoplasmic cytochrome $c$ in populations that do not express GFP-cytochrome $c$.

\section{Method}

Cells $\left(1 \times 10^{5}\right)$ were harvested and treated with $100 \mu \mathrm{l}$ digitonin $(50 \mu \mathrm{g} / \mathrm{ml}$ in PBS with $100 \mathrm{mM} \mathrm{KCl})$ for $5 \mathrm{~min}$ on ice (until $>95 \%$ were permeabilized as assessed by trypan blue exclusion). Cells were fixed in paraformaldehyde (4\% in PBS) for $20 \mathrm{~min}$ at room temperature, washed three times in PBS and incubated in blocking buffer (3\% BSA, $0.05 \%$ saponin in PBS) for $1 \mathrm{~h}$. The cells were incubated overnight at $4^{\circ} \mathrm{C}$ with $1: 200$ anticytochrome $c$ monoclonal antibody (clone 6H2.B4, BD Pharmingen, San Diego, CA, USA) in blocking buffer, washed three times and incubated for $1 \mathrm{~h}$ at room temperature in $1: 200$ PE-labeled secondary antibody (Silenus, Hawthorn, Australia) in blocking buffer. The cells were then analyzed by flow cytometry detecting PE fluorescence in FL-2. Cells were visualized by fluorescence microscopy to confirm the FACS analysis. When Alexa 488 (Molecular Probes, Eugene, OR, USA) conjugated to the $6 \mathrm{H} 2 . \mathrm{B} 4$ anticytochrome $c$ antibody was used, the secondary antibody step was not required. Note, $\mathrm{Ca}^{2+}$ will induce permeability transition in the mitochondria of permeabilized cells. EDTA should therefore be added to the digitonin permeabilization buffer if the cells were treated in media containing high $\mathrm{Ca}^{2+}$.

To test our hypothesis, we treated Jurkat T-cell leukemia cells with dimeric (leucine zipper) TNF-related apoptosisinducing ligand (TRAIL) for $4 \mathrm{~h}$. The cells were harvested and 
a

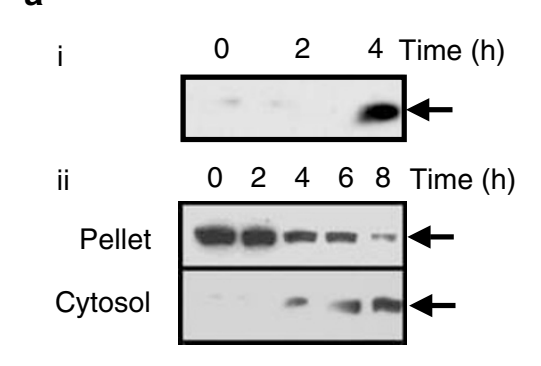

b

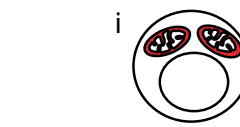

Healthy cell

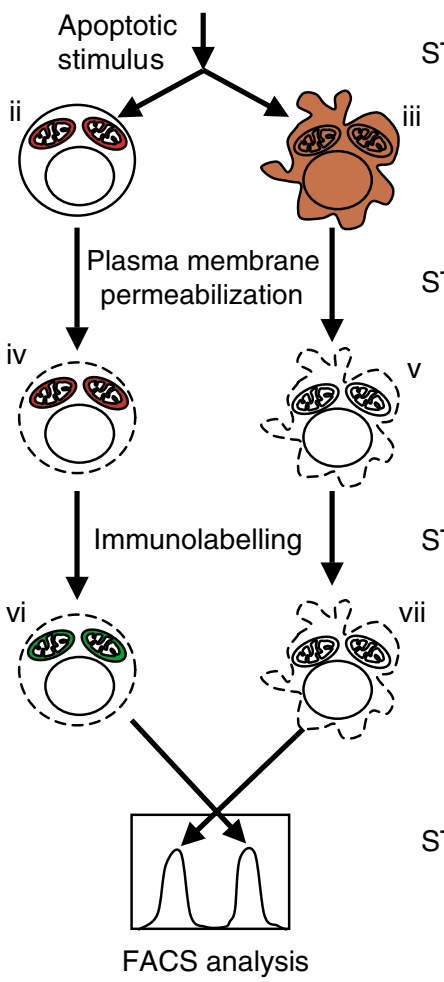

C

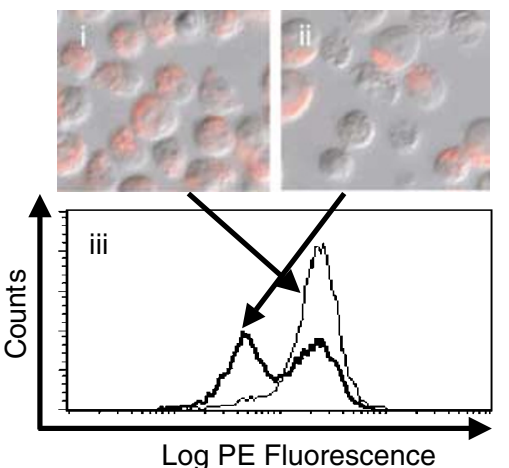

LOg PE Fluorescence

c

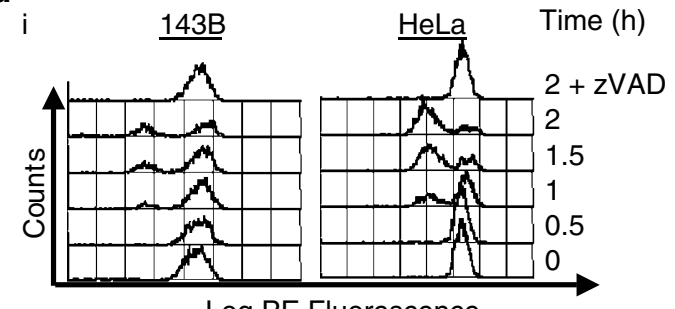

Log PE Fluorescence

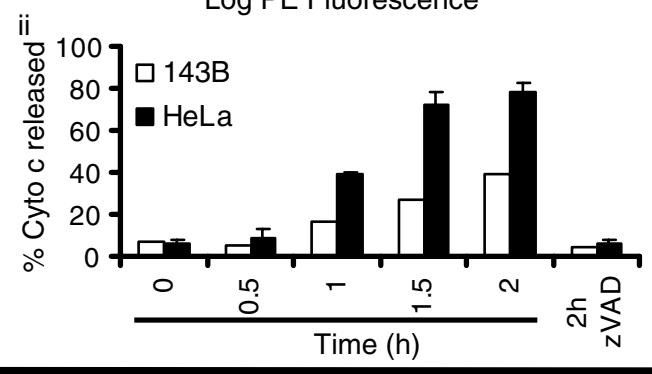

e

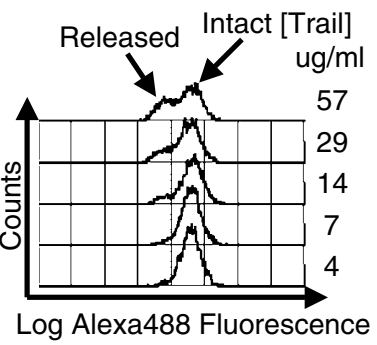

Figure 1 (a) Western blot analysis of cytochrome $c$ in Jurkat cell extracts isolated by selective permeabilization of the plasma membrane. ${ }^{4}$ (i) Cytochrome $c$ detected in cytosolic extracts from Jurkat cells treated with $20 \mu \mathrm{M}$ etoposide for 0,2 and $4 \mathrm{~h}$. (ii) Cytochrome $c$ detected in cytosolic and pellet (containing mitochondria) extracts from Jurkat cells treated with $20 \mu \mathrm{M}$ etoposide for 0, 2, 4, 6 and $8 \mathrm{~h}$. Arrows indicate cytochrome $c$. (b) Key steps in the protocol described in this letter. Step 1. (i) Healthy cells have mitochondrially localized cytochrome $c$ (red). Following treatment with an apoptotic stimulus, (ii) cells that have not undergone mitochondrial outer membrane permeabilization retain cytochrome $c$ in the mitochondria, while (iii) cytochrome $c$ diffuses through cells in which mitochondrial outer membrane permeabilization has occurred. Step 2. Selective permeabilization of the plasma membrane with digitonin allows virtually all of the cytoplasmic cytochrome $c$ to diffuse out of the cells. (iv) Cytochrome $\mathrm{c}$ remains in cells with intact mitochondria, but $(\mathrm{v})$ is absent from cells that have undergone mitochondrial outer membrane permeabilization. Step 3. The cells are then fixed and stained by immunocytochemistry. (vi) Cells with intact mitochondria will stain positive for cytochrome $c$ (green), while (vii) cells in which cytochrome $c$ was released from the mitochondria will have low fluorescence. Step 4. FACS analysis of the cells can then be used to count the cells with high (intact mitochondria) and low (mitochondria that have released cytochrome c) fluorescence. (c) Jurkat cells were treated with $230 \mathrm{ng} / \mathrm{ml}$ TRAlL and harvested after $4 \mathrm{~h}$. Cytochrome $c$ translocation was assessed by selective permeabilization of the plasma membrane followed by immunocytochemistry and (i and ii) fluorescence microscopy or (iii) FACS. Panels (i) and (iii) (thin line) represent untreated cells. Panels (ii) and (iii) (thick line) represent treated cells. (d) HeLa cells and 143B cells were treated with $230 \mathrm{ng} / \mathrm{ml}$ TRAIL and harvested at the times indicated. (i) Cells were analyzed by FACS for cytochrome $c$ content following plasma membrane lysis and immunocytochemistry. (ii) The number of cells with low fluorescence (cells in which cytochrome $c$ was released during apoptosis) were quantitated. (e) HeLa cells were treated with various concentrations of TRAIL and harvested after $2 \mathrm{~h}$. The plasma membranes were selectively lysed. Cells were stained with Alexa 488 conjugated anticytochrome $c$ and analyzed by FACS

assayed for cytochrome $c$ release as described above. We found that untreated Jurkat cells had high fluorescence as detected by fluorescence microscopy (Figure 1ci) and flow cytometry (Figure 1ciii, thin line). In Jurkat cells treated with TRAIL, a significant number of cells had low fluorescence (indicating loss of cytochrome $c$ from the mitochondria) as 
Table 1 Advantages and disadvantages of techniques used to measure cutochrome c translocation

\begin{tabular}{|c|c|c|}
\hline Technique & Advantage & Disadvantage \\
\hline $\begin{array}{l}\text { Homogenization or } \\
\text { nitrogen cavitation }\end{array}$ & $\begin{array}{l}\text { Standard technique for cell } \\
\text { fractionation }\end{array}$ & $\begin{array}{l}\text { Laborious } \\
\text { Not all cells are disrupted } \\
\text { Over zealous disruption leads to high background } \\
\text { High numbers of cells needed } \\
\text { Quantitation is difficult }\end{array}$ \\
\hline $\begin{array}{l}\text { Digitonin/sterptolysin } \\
\text { O permeabilization }\end{array}$ & $\begin{array}{l}\text { Assays most cells in a population } \\
\text { Relatively small number of cells needed }\end{array}$ & Quantitation is difficult \\
\hline Immunocytochemistry & $\begin{array}{l}\text { Visual confirmation } \\
\text { Small number of cells needed }\end{array}$ & $\begin{array}{l}\text { Difficult to determine release in suspension or round cells } \\
\text { Subjective } \\
\text { Counting is laborious }\end{array}$ \\
\hline Cytochrome $c$-GFP & $\begin{array}{l}\text { Can follow translocation in live cells } \\
\text { Can be easily quantitated }\end{array}$ & $\begin{array}{l}\text { Follows an overexpressed fusion construct rather } \\
\text { than endogenous protein } \\
\text { Limited to a small number of cells engineered to } \\
\text { express this construct }\end{array}$ \\
\hline
\end{tabular}

detected by fluorescence microscopy (Figure 1cii) and flow cytometry (Figure 1ciii, thick line). Similarly, we found cells with high fluorescence (intact mitochondria) and low fluorescence (cytochrome $c$ released) in TRAIL-treated 143B osteosarcoma cells and HeLa cervical carcinoma cells (Figure $1 \mathrm{di})$. In each case, the apoptotic and nonapoptotic populations were sufficiently distinct to allow quantitation of each population (Figure 1dii). A time course study revealed that the number of cells with cytoplasmic cytochrome $c$ increased with time. This confirmed our previous results using Cc-GFPexpressing HeLa cells where we showed that individual cells release cytochrome $c$ at different times. ${ }^{5}$ The absence of an intermediate peak also corroborated our previous findings that cytochrome $c$ release was rapid and complete. ${ }^{5}$

Using an alexa 488-conjugated cytochrome $c$ antibody, we found that we could omit the secondary antibody step and still quantitate the number of cells with intact mitochondria or cytoplasmic cytochrome $c$. While this shortened the assay time considerably, the difference in fluorescence intensity of control cells and cells that have released cytochrome $c$ was not as distinct as when we used the labeled secondary antibody (Figure 1e).

\section{Concluding remarks}

Determining whether cytochrome $c$ release occurs during various types of cell death is a standard experiment in the field, and there is considerable interest in determining the mechanism of cytochrome $c$ release and its regulation by $\mathrm{Bcl}$ 2 family members. While current techniques offer valid assays for cytochrome $c$ release during apoptosis, we believe that the ability to rapidly and nonsubjectively quantitate the number of cells that have cytoplasmic cytochrome $c$ will significantly enhance our understanding of this event. This rapid assay can be used on a variety of adherent and nonadherent cell lines. Preliminary results have shown that this assay can also be used on primary cells, and it may be possible to use this assay on isolated mitochondria. It remains unclear whether this assay can be used for measuring the release of less abundant proteins, such as SMAC/Diablo from the mitochondrial intermembrane space.

\section{Acknowledgements}

This work was supported by a Peter Doherty Fellowship from the National Health and Medical Research Council Australia (REG Key 165405) and a post-doctoral award from AMRAD Australia to NJW. JAT is a Principal Research Fellow of the National Health and Medical Research Council, Australia. Leucine zipper TRAIL was a kind gift from Immunex Corporation (Seattle, WA, USA). We thank Mark Smyth and the Cancer Immunology Research unit at the Peter MacCallum Cancer Institute for discussions.

\footnotetext{
NJ Waterhouse ${ }^{*, 1}$ and JA Trapani ${ }^{1}$

${ }^{1}$ Research Division, Peter MacCallum Cancer Institute, Locked Bag 1, A'Beckett Street, Melbourne, Vic 8006, Australia

* Corresponding author: NJ Waterhouse, Tel: +61 39656 3725; Fax: 6139656

1411; E-mail: nigelwaterhouse @ hotmail.com

1. Martinou JC et al. (2001) Nat. Rev. Mol. Cell. Biol. 2:63-67.

2. Waterhouse NJ et al. (2002) Biochimie 84:113-121.

3. Cain K et al. (2002) Biochimie 84:203-214.

4. Waterhouse NJ et al. (2001) Methods Cell Biol. 66:365-391

5. Goldstein JC et al. (2000) Nat. Cell Biol. 2:156-162.
} 